\title{
Permeation through Lipid Bilayers by Adhesion of Giant Vesicles on Decorated Surfaces
}

\author{
A.-L. Bernard, ${ }^{* \dagger}{ }^{\dagger}$ M.-A. Guedeau-Boudeville, ${ }^{\dagger}$ O. Sandre, ${ }^{\ddagger}$ S. Palacin, ${ }^{\S}$ \\ J.-M. di Meglio," and L. J ullien ${ }^{\perp}$
}

Laboratoire de Physique de la Matière Condensée CNRS URA 792, Collège de France, 11 place Marcel in Berthel ot, 75231 Paris Cedex 05, France, Physico Chimie Curie, CNRS UMR 168, Section de Recherche de I'Institut Curie, 11 rue P. et M. Curie, F-75231 Paris Cedex 05, France, Service de Chimie Moléculaire, CEA Saclay, Bât. 125 F-91191 Gif sur Yvette Cedex, France, University Louis Pasteur and Institut Universitaire de France, Institut Charles Sadron CNRS UPR 022, 6 rue Boussingault, 67083 Strasbourg Cedex, France, and Département de Chimie CNRS UMR 8640, Ecole Normale Supérieure, 24 rue Lhomond, 75231 Paris Cedex 05, France

\section{Received October 13, 1999}

\begin{abstract}
We describethe dynamics of interaction of giant vesicles with properly designed decorated surfaces. The spreading of the vesicles leads to major deformations and is accompanied by the creation of transient macroscopic pores in the micrometer range. Different phenomena that have been observed by changing either the characteristic scale or the geometry of the decorated surfaces are reported and discussed.
\end{abstract}

\section{Introduction}

Controlling the transport of water-soluble solutes through lipid bilayers is a major issue in developing new approaches in drug targeting and delivery. Many strategies have already been designed to enhance the transmembrane passage of species as small as ions or as large as proteins. Chemical strategies generally make use of the addition of a suitable agent (detergent, carrier, channel, ...) whose conformation and favorable partition between theaqueous phaseand the bilayer is responsible for the increase of permeability of the lipid barrier. In these strategies, transport is expected to occur in a steady state as long as the promoting factor is present in the system. Despite encouraging results obtained by acting upon thepermanent endocytotic processes for transporting the largest solutes such as DNA, ${ }^{1,2}$ chemical strategies aremost often restricted tothetransport of small species. ${ }^{3}$ Physical strategies consist in applying an external perturbati on (temperature rise, ${ }^{4}$ pul ses of electrical field ${ }^{5}$...) which leads to the formation of large permeating defects or pores in thebilayer. U nfortunately, together with often severe practical requirements, the physical approaches are intrinsically limited by the relaxation time of return to thenonper meable equili briumstate via lipid mi gration or flip-flop processes for instance. Indeed, this characteristic relaxation time determines a kinetic window for permeation, in terms of both its extent and duration; the

* To whom correspondence should be addressed. Present address: L'Oréal, Service d'Appui Physico-Chimie, 66 rue Henri Barbusse, 92117 Clichy cedex, France. E-mail: albernard@recherche. Ioreal.com.

† URA 792, Collège de France.

‡ UMR 168, Physico Chimie Curie.

$\S$ Service de Chimie Moléculaire, CEA Saclay.

II UPR 022, Institut Charles Sadron.

$\perp$ UMR 8640, Ecole Normale Supérieure.

(1) Behr, J.-P. Acc. Chem. Res. 1993, 26, 274

(2) Ledley, F. D. Hum. Gene Ther. 1995, 6, 1129.

(3) Cevc, G. Phospholipid handbook; Cevc, G., Ed.; Marcel Dekker, 1993; pp 318-321.

(4) Lasic, D. D. Liposomes: from Physics to Applications; Elsevier Science Publishers: Amsterdam, 1993; pp 318-321.

(5) Zhelev D.; Needham, D. Biochim. Biophys. Acta 1993, 1147, 89 Moroz J .; Nelson, P. Biophys. J . 1997, 72, 2211. permeation occurs under kinetic control in analogy with the kinetic/thermodynamic controls of wide use in chemistry. ${ }^{6}$ In the continuity of our previous studies devoted to the setup of chemical ${ }^{7}$ and physical ${ }^{8,9}$ strategies for inducing solutetransport through lipid bilayers, wehave been concerned with a new approach making use of a kinetic control that should lead to the transport of the largest solutes. Its basic principle lies in inducing the formation of transient pores in the membrane of giant vesicles ${ }^{10,11}$ adsorbed on suitably decorated surfaces interacting with the membrane.

In this work, the interaction between vesicles and substrates originates from thestrong and fast ${ }^{12}$ adhesion of a charged bilayer onto a surface of opposite charge. Anticipating that a contrast between al ternating more or less adhesive elements of surface should facilitate permeation, attention has been focused on decorated surfaces with a surface fraction $x_{S}$ of the more adhesive portions $\left(x_{s}=0.5\right.$, Figure 1$)$. We have examined the significance of the scale and of the geometry of the surface patterns. In view of the bilayer thickness (a few nanometers) and of thetypical radius of giant vesicles $(10-100 \mu \mathrm{m})$, patterns with periodicities ranging from 1 to $50 \mu \mathrm{m}$ have been chosen. Hence, thecharacteristicsizeof the pattern should be(i) largeenough to promotelargemorphological changes of the vesicle and (ii) small enough to get a permeation

(6) J ullien, L.; Proust, A.; Le Menn, J .-C. J . Chem. Educ. 1998, 75, 194.

(7) Pregel, M. J .; J ullien, L.; Canceill, J .; Lacombe, L.; Lehn, J .-M J. Chem. Soc., Perkin Trans. 2 1995, 417 and references therein.

(8) Dvolaitzky, M. de Gennes, P. G.; Guedeau, M.-A.; J ullien, L. C. R. Acad. Sci., Ser. II 1993, 316, 1687.

(9) Guedeau-Boudeville, M.-A.J ullien, L. di Meglio, J .-M. Proc. Natl. Acad. Sci. 1995, 92, 9590.

(10) Menger F. M.; Angelova, M. I. Acc. Chem. Res. 1998, 31, 789.

(11) Needham D.; Zhelev, D. V. Surfact. Sci. Ser. 1996, 62, 373.

(12) "fast" qualification deserves a comment. To be effective for permeation, the adhesion must occur faster than the relaxation of the bilayer tension. In contrast to more specific interactions requiring the appropriatepositioning of interacting chemical groups, theel ectrostatic interaction arising between two surfaces of opposite charge leads to adhesion as soon as their relative distance drops bel ow a characteristic screening length depending on the experimental conditions. See for instance: Northrup, S. H.; Boles, J. O.; Reynolds, J. C. L. J . Phys. Chem. 1987, 91, 5991 

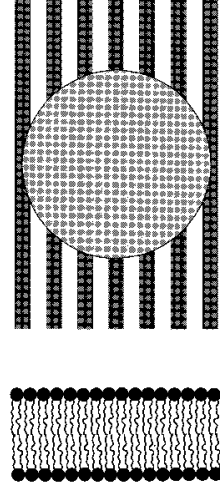

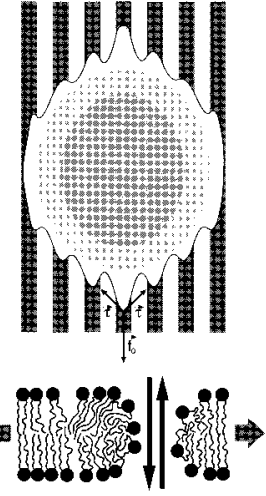

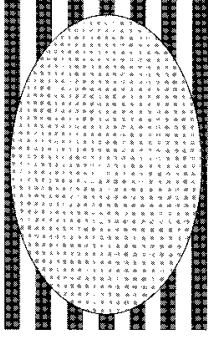

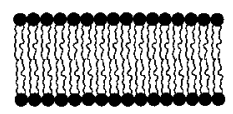

C
Figure 1. Forcing permeation through lipid bilayers by membrane adhesion on decorated attractive surfaces. Striped patterns have been chosen for the illustration with $x_{s}=0.5$. From left to right: (A) nonadhesive regime, the vesicle stays just abovethesurface, the bilayer is not stretched and exhibits a low permeability toward water-soluble species; (B) dynamic regime of spreading, the vesicle spreads out onto the surface and adapts its shape to decrease the system energy, in this regimethebilayer becomesstrained and its permeability toward hydrophil icsol utes is increased as a consequence of thecreation of microscopic defects or of macroscopic pores; (C) regime of equilibrium. Several mechanisms (lipid migration, lipid flipflop, ...) lead to the partial relaxation of the membranetension. The defect density drops and the pores close.

that does not depend on the size and position of the vesicle. Stripe, square, triangle, and herringbone geometrical shapes have been retained. In addition to promote permeation, we are also interested in highlighting (i) the dynamics of vesiclespreading onto decorated surfaces with respect to the results obtained on smooth surfaces. ${ }^{13}$ Despite the extensive use of vesicles for preparing supported bilayers and the numerous theoretical reports on the equilibrium morphology of vesicles interacting with surfaces, ${ }^{14}$ the dynamic aspect has been rarely studied, ${ }^{15}$ (ii) the most favorable patterns for promoting (or hindering) vesicle adhesion by changing the characteristic lengths and symmetries with regard to the thermal fluctuations of the vesicles, ${ }^{16,17}$ and (iii) any vesiclemotility driven by Marangoni effect on suitably decorated surfaces. ${ }^{18}$

\section{Experimental Section}

Giant vesides have been prepared by swelling a thin film obtained after evaporation of freshly prepared chloroform/ methanol 9:1 solutions of EPC (egg phosphatidylcholine, SigmaAldrich) in $50 \mathrm{mM}$ sucrose aqueous solutions in the presence of an alternating el ectrical field. ${ }^{19}$ Toinducethesedimentation onto the surface, the vesicles are transferred at constant osmolarity into a less dense aqueous solution ( $50 \mathrm{mM}$ glucose). ${ }^{20}$ It has been experimentally observed that the trace amounts of negatively charged species resulting from EPC hydrolysis (evaluated at about $0.1 \% \mathrm{~mol} / \mathrm{mol}^{21}$ ) are sufficient to promote vesicleadhesion

(13) Bernard, A.-L.; Guedeau-Boudeville, M.-A.;J ullien, L.; di Meglio, J.-M. Strong Adhesion of Giant Vesicles on Surfaces: Dynamics and Permeability. Langmuir 2000, 16, 6809.

(14) Seifert U.; Lipowsky, R. Langmuir 1991, 7, 1867. Seifert U . Lipowsky, R. Mol. Cryst. Liq. Cryst. 1991, 202, 17.

(15) Baumgärtner A.; Bhattacharjee, S. M. J . Chem. Phys. 1997, 107,4390

(16) Brochard F.; Lennon, J .-F. J . Phys. (Paris) 1975, 36, 1035.

(17) Singhvi R.; et al. Science 1994, 264, 696.

(18) Durand, I.; J önson, P.; Misbah, C.; Valance, A.; Kassner, K. Phys. Rev. E 1997, 4, 56.

(19) Angelova, M. I. Mol. Cryst. Liq. Cryst. 1987, 152, 89

(20) Gravity does not here play a significant role on the permeation processes. See for instance: Kraus, M.; Seifert, U.; Lipowsky, R Europhys. Lett. 1995, 32, 431. to the positively charged surfaces. The decorated surfaces have been obtained using the microcontact printing technique introduced by Whitesi des.22 PDMS (poly(dimethylsiloxane)) patterned stamps impregnated with a $1 \mathrm{mM}$ ethanol solution of dodecanethiol were applied onto ITO (indium tin oxide)-coated glass surfaces covered by a thin optically transparent gol d layer. After removal of thestamp, the resulting surface was stepwise dipped for $1 \mathrm{~min}$ into a $1 \mathrm{mM}$ water/ethanol 1:9 solution of sodium 3-mercaptopropane sulfonate, then cautiously rinsed with Millipore water and finally immersed for $24 \mathrm{~h}$ in a $0.01 \%$ aqueous solution of positively charged poly-L-lysine (MW $=150000$ 300000, Sigma-Aldrich). The decorated surfaces are then immediately used after extensive washing with Millipore water. Selective etching processes and atomic force microscopy (AF M) imaging in friction mode have been used to characterize the decorated surfaces. They have shown that the patterns have been correctly transferred from the PDMS stamps to the gold surface, producingalternating hydrophilicand hydrophobicareas on the surface. The patterns have been al so directly observed by reflection interference contrast microscopy (RICM).23 The spreading of the giant vesicles onto the decorated surfaces as well as induced bilayer permeability have been examined using a custombuilt microscope working in either phase contrast (to determine the vesicle radius) or evanescent wave induced fluorescence (EWIF).13,24 In thelatter case, a suitable fluorescent water-soluble dye (calcein) was added to the external vesicular pool; it becomes thus possibletovisualizethecontact zone between thespreading vesicleand the decorated surfaces. EWIF microscopy alsoallows the determination of the shape of the adsorbed vesicle at the micrometer scale.13,24 In addition, one can evidence any permeability of themembranetoward thefluorescent probeby analyzing the contrast of the vesicle (dark) with regard to the background (bright). Complementary experiments have been performed with RICM or with epifluorescence microscopy using a lipophilic aminostyryl pyridininium dye (S-467, M olecular Probes). These techniques enablestudy of thetopology of thebilayer with a high resolution.

\section{Results}

Figures 2, 3, 4, and 5 display the typical behavior of an EPC giant vesiclespreading onto surfaces patterned with stripes (Figure2), herringbone(Figure3), squares (Figure 4) and grids (Figure 5) exhibiting different attraction strengths for EPC vesicles. The initial time is taken as the moment when the vesicle sedimenting toward the decorated surfaces stops its descent. From this time, three different regimes havebeen evidenced. Thelongest regime (regime I; characteristic time $\tau_{1}$ in the minute range) is associated with the formation of a first contact point between the bilayer and the surface. By comparison of the behavior on stripes of different widths $(2.5,5$, and 12 $u \mathrm{~m}), \tau_{1}$ is shown to increase when the surface fraction of attractivedomains $x_{s}$ is reduced. N evertheless, $\tau_{1}$ remains in thesamerange of values as observed for smooth surfaces under comparable conditions. ${ }^{13}$ By contrast to the case of smooth surfaces, the second regime reveals by EWIF microscopy a long sequential process of spreading (regime II, characteristictime $\tau_{2}$ in therange of 10 s to becompared with 50 ms on smooth surfaces). Thevesi clefirst undergoes a local spreading; for instance, it spreads rapidly al ong a stripe (Figure 2B). Then, one observes spreading along a neighboring attractive stripe (Figure $2 \mathrm{C}$ ). This growth process occurs repeatedly until part of a homogeneous adhering surface of the vesicle is observed (Figures 2E, 3E, and 4B). This process looks analogous to the $\mathrm{H}$ aines jump of a liquid on a corrugated surface. ${ }^{25}$ The adhered

(21) Pincet, F.; Cribier, S.; Perez, E. Eur. Phys. J . 1999, 311, 127

(22) Whitesides, G. Angew. Chem., Int. Ed. 1998, 37, 550.

(23) Rädler, J ., Sackmann, E. J . Phys. II 1993, 3, 727.

(24) Bernard, A.-L.; Guedeau-Boudeville, M.-A.;j ullien, L.; di Meglio, J .-M. Europhys. Lett. 1999, 46 (1), 101.

(25) Haines, W. G. J . Agric. Sci. 1930, 20, 97. 

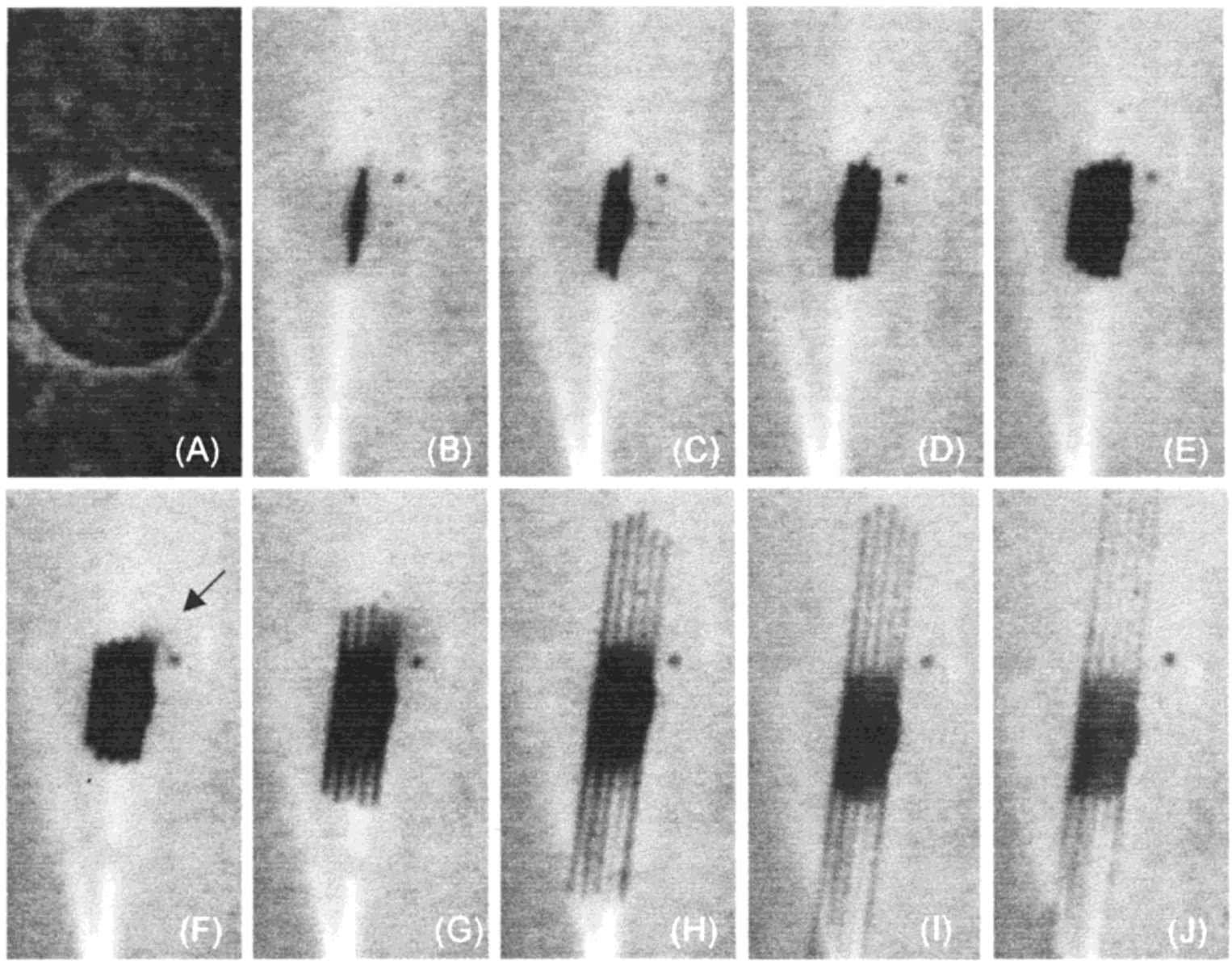

Figure 2. Spreading of an EPC vesicle on striped patterns (width $=2.5 \mu \mathrm{m}$ ) as a function of time. Vesicle as observed in phase contrast microscopy at $\mathrm{t}=0(\mathrm{~A})$. Vesicle as observed in EWIF microscopy (the depth of penetration of the evanescent wave is estimated between $600 \mathrm{~nm}$ and $1 \mu \mathrm{m})$ at $t=1(\mathrm{~B}), 3(\mathrm{C}), 4(\mathrm{D}), 15(\mathrm{E}), 67(\mathrm{~F}), 68(\mathrm{G}), 73(\mathrm{H}), 84(\mathrm{I})$, and $92 \mathrm{~s}(\mathrm{~J})$. The arrow on photograph $\mathrm{F}$ emphasizes the formation of a macroscopic pore along the contact line.

part of the vesicle appears homogeneously dark in EWIF microscopy, and the intensity of the vesicle image does not evolve during the second regime. The vesicle shape remains circular as observed by phase-contrast microscopy. Then, the vesicle pauses for a period equal to a few seconds on square and grids patterns (Figures 4 and 5) or to 1 min on striped and herringbone patterns (Figures 2 and 3). The last regime (regimellI, characteristic time $\tau_{3}$ in the minute range) begins with the formation of a pore in the lipid bilayer along the contact line. In EWIF microscopy, the pore is evidenced by the emission of a diffuse dark cloud (see arrow in Figures 2F, 3F, 4C, and $5 E$ ). Finally one notices spreading of thelipid bilayer al ong the attractive zones at a constant velocity of about 20 $\mu \mathrm{m} \cdot \mathrm{s}^{-1}$. Epifluorescence microscopy has been used to demonstrate that the vesicle parts spreading along attractive lines are not simply adsorbed bilayers but true tubular extensions (pseudopods) of the vesicle undergoing a Rayleigh-type instability (Figure 6A). ${ }^{26}$ In view of the difference in luminosity between attractive and nonattractivestripes, EWI F microscopy confirms the preceding observation. Indeed, the tubes lower the fluorescence intensity abovetheattractivestripes in EWIF microscopy (Figure6B). EWIF alsoshows that the vesicle/background optical contrast drops during regimellI . At the end of the spreading process, the vesicleadopts a shapethat depends on the surface pattern. In the case of the $2.5 \mu \mathrm{m}$ wide striped pattern, phase contrast microscopy shows that the vesicle is elongated along the stripe axis (F igure 6C).

(26) Bar-Ziv, R.; Moses, E. Phys. Rev. Lett. 1994,73, 1392.
The spindle adopted morphology is confirmed by RICM (Figure 6D) and epifluor escence microscopy (F igure 6E ). A typical contact angle of the vesicle along the in-plane perpendicular axis to the stripes equal to $15-20^{\circ}$ was extracted from the spacing between the interference fringes observed by RICM. Among the different regimes observed, regime III is the only one to exhibit scale- and geometry-dependent features. Within theseries of striped patterns, regime III is only present for patterns with the $2.5 \mu \mathrm{m}$ periodicity. For patterns made of larger stripes at the same value of $X_{s}$, (i) the spreading phenomenon stops at the end of regimell (regime III does not occur) and (ii) no significant solute permeation can be observed and the vesicular shapeas observed by phase-contrast microscopy remains circular. Note that under the experimental conditions investigated, the vesicle never blows up on stripepatterns. In contrast, regimell I was al ways present on the other decorated surfaces examined (triangles, squares, grids, herringbone) and led to solute permeation. Moreover, the spreading vesicles could not be observed anymore by phase contrast, and it is thus suggested that the latter geometries promote vesicle rupture.

No motion of the center of mass of vesicles has been ever noticed during experiments, even when a driving force was expected (largetriangleand square patterns for example). But an oscillating behavior has been frequently observed when aged patterned stamps of $2.5 \mu \mathrm{m}$ stripes were used instead of the new stamps that were used to obtain the results reported in the preceding paragraph. Under such conditions, E PC vesicles hardly spread during 

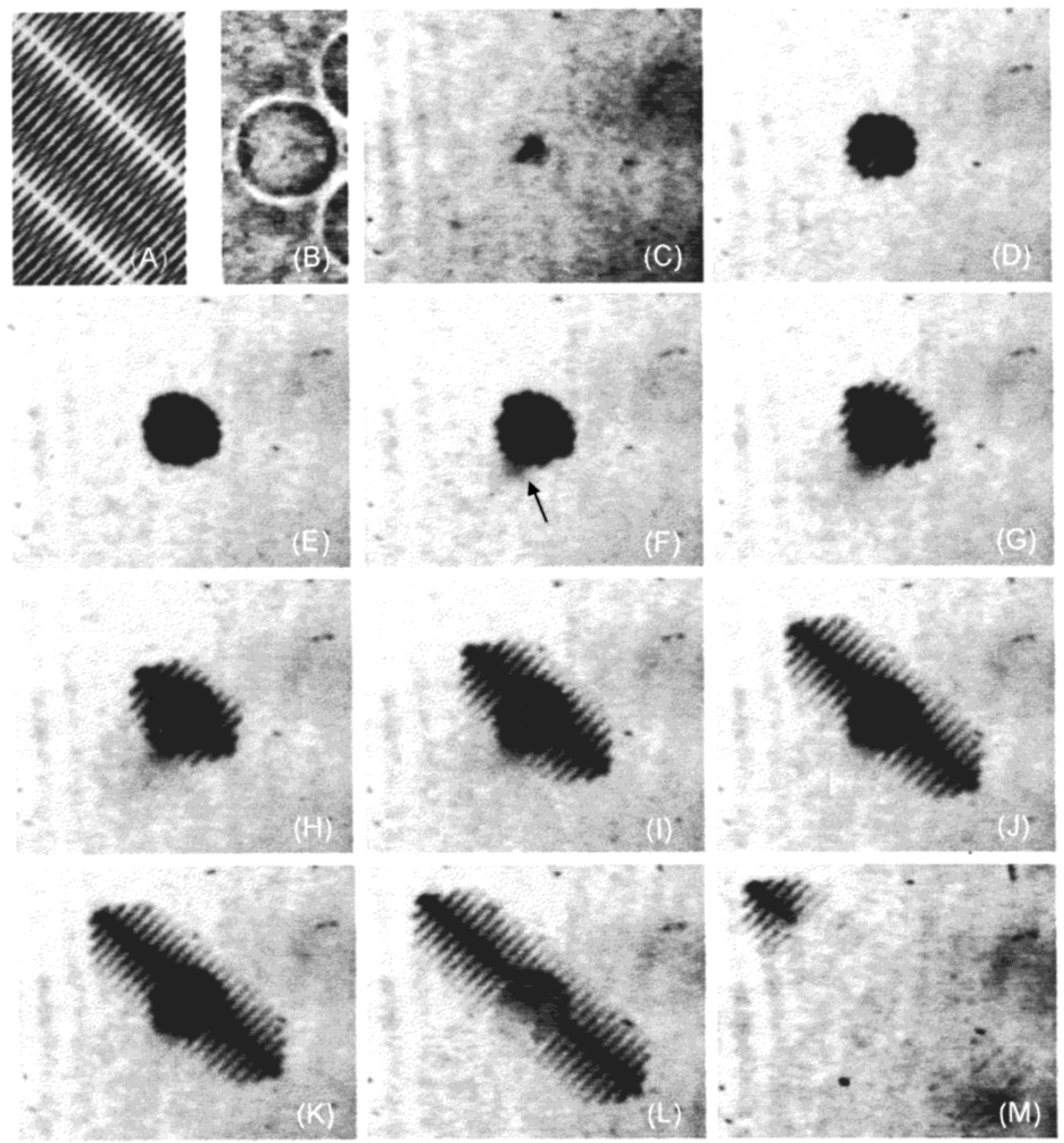

Figure 3. Spreading of an EPC vesicle on herringbone patterns (width $=50 \mu \mathrm{m}$ ) as a function of time. Patterning stamp as observed by phase contrast microscopy (A). Vesicle as observed in phase contrast microscopy at $t=0$ (B). Vesicle as observed in $E$ WIF microscopy (the depth of penetration of the evanescent wave is estimated between $600 \mathrm{~nm}$ and $1 \mu \mathrm{m})$ at $t=1(C), 6(D), 31(E)$, $31.3(\mathrm{~F}), 32(\mathrm{G}), 33(\mathrm{H}), 35(\mathrm{I}), 37(\mathrm{~J}), 39(\mathrm{~K}), 40(\mathrm{~L})$, and $43 \mathrm{~s}(\mathrm{M})$ ). The arrow on picture $\mathrm{F}$ emphasizes the formation of a macroscopic pore along the contact line.

regimell I but oneobserves theregular openingand cl osing of a pore formed on the contact line at the bor der between zones exhibiting different attractive interactions (Figure 7). At the end of this beating step, one notices a loss of vesicle contrast in EWIF microscopy which is evidence of the internalization of the fluorescent probe.

\section{Discussion}

Our observations emphasize the complexity of the spreading behavior of giant vesicles on adhesive surfaces. Similar to the case of smooth surfaces, ${ }^{13}$ a sequential dynamic process with three regimes has been observed (Figure 8).

Regimel can bedescribed as the approach regime. After the sedimentation process controlled by buoyant and viscous forces, the vesiclemotion considerably slows down as soon as the distance between veside and surface becomes small enough (comparable to the membrane fluctuations). Thevesi clethen appears immobile in phase contrast microscopy. The corresponding time scale $\tau_{1}$ is the longest time encountered during the entire adhesion process. In the case of undecorated surfaces which sufficiently attract the lipid bilayer, ${ }^{13}$ spreading begins as soon as a contact between the membraneand thesurface is established. In the case of decorated surfaces, one also has to take into account the probability of creating a nucleation point whose stabilizing energy exceeds the energy of thermal fluctuations $\left(\mathrm{k}_{\mathrm{B}} \mathrm{T}\right)$. I $\mathrm{n}$ therange of scales investigated, this probability is governed by the surface fraction of attractivezones. Nevertheless, it is reasonable to anticipate that some more complicated dependence of $\tau_{1}$ on the pattern geometry of the decorated surface may 


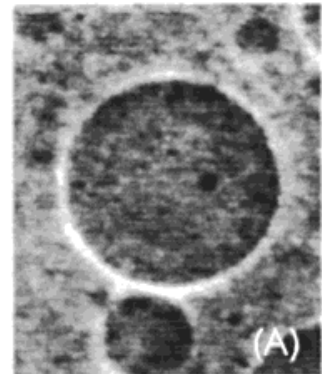

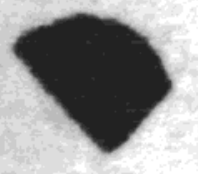

(B)
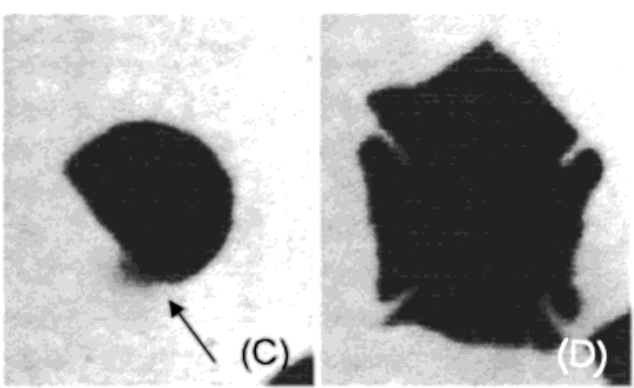

Figure 4. Spreading of an EPC vesicle on square patterns (width of the square grid, $5 \mu \mathrm{m}$; side of the attractive square, $50 \mu \mathrm{m}$ ) as a function of time. Vesicle (diameter $50 \mu \mathrm{m}$ ) as observed in phase contrast microscopy at $t=0$ (A). Vesicle as observed by EWIF microscopy (the depth of penetration of the evanescent wave is estimated between $600 \mathrm{~nm}$ and $1 \mu \mathrm{m})$ at $t=5(B), 30(C), 34(D)$, and $35 \mathrm{~s}(\mathrm{E})$. The arrow on picture $\mathrm{C}$ emphasizes the formation of a macroscopic pore along the contact line.
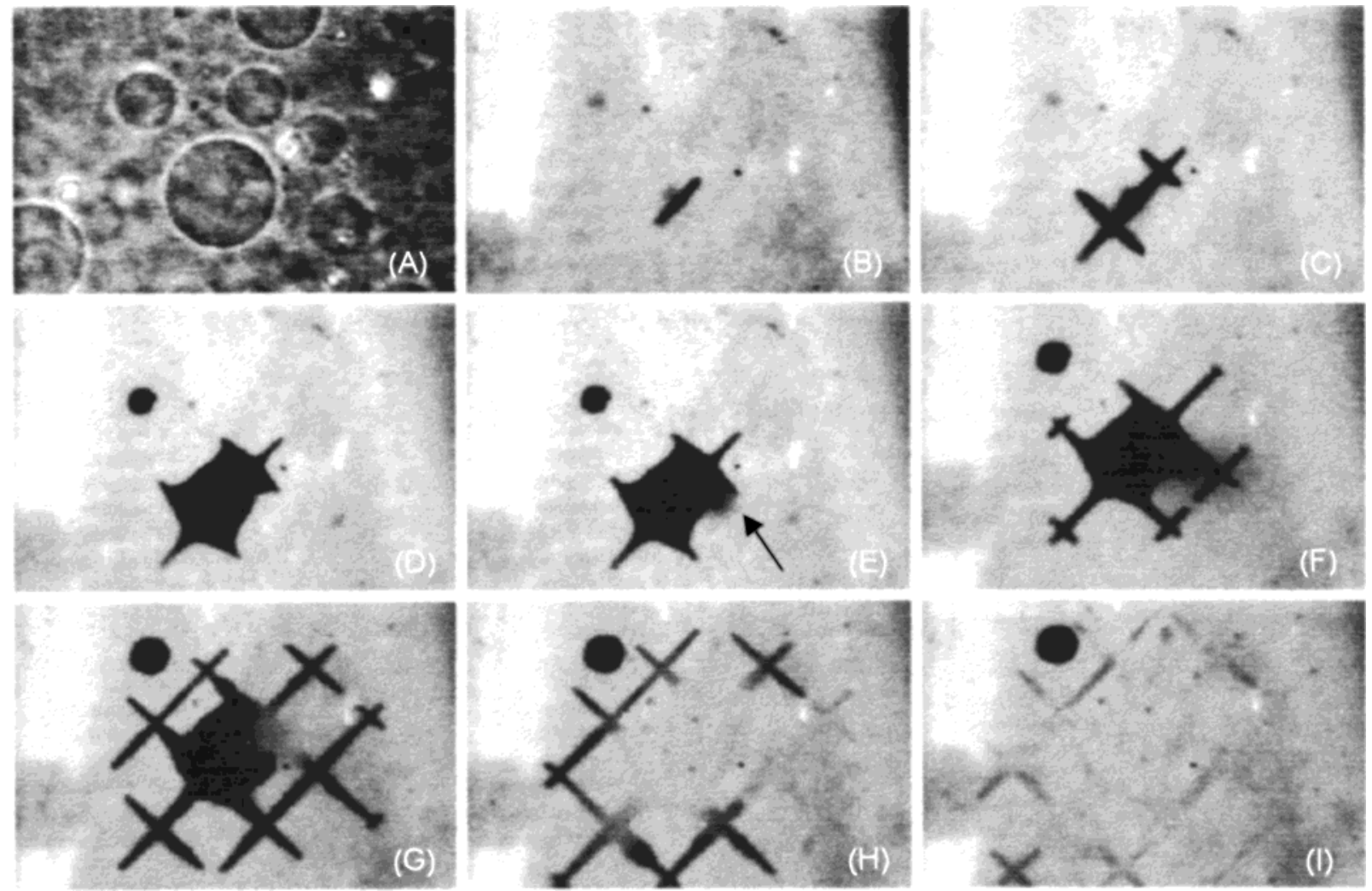

Figure 5. Spreading of an EPC vesicle on grid patterns (width of the attractive square grid, $5 \mu \mathrm{m}$; side of the square, $50 \mu \mathrm{m}$ ) as a function of time. Vesicle ( $50 \mu \mathrm{m}$ diameter) as observed by phase contrast microscopy at $\mathrm{t}=0$ (A). Vesicle as observed by EWIF microscopy (the depth of penetration of the evanescent wave is estimated between $600 \mathrm{~nm}$ and $1 \mu \mathrm{m})$ at $\mathrm{t}=0.3(\mathrm{~B}), 2.7(\mathrm{C}), 24.5$ (D), $25.3(\mathrm{E})$, $28.1(\mathrm{~F}), 34.9(\mathrm{G}), 45.9(\mathrm{H})$, and $47 \mathrm{~s}(\mathrm{I})$. The arrow on picture $E$ emphasizes the formation of a macroscopic pore al ong the contact line.

exist but at a smaller length scale than that investigated in thepresent study. A crudeestimate of thetypical length scale at which such effects should manifest themselves can be obtained as follows. In the preceding model used to derive $\tau_{1}$, the assumption of a planar bilayer has been used. In fact, a fluctuating vesicle at a distance $h$ of a planar surfacecan bedescribed as thesum of independent elements whose characteristic lengths are $\xi_{\|}=\left(\kappa_{d} / \mathrm{kTh}\right)^{1 / 2}$ and $\xi_{\perp} \approx \mathrm{h}$, where $\kappa_{\mathrm{c}}$ designates the Helfrich bending modulus. ${ }^{27}$ If the surface is made of zones in the $\xi_{\|}$scale range (about $600 \mathrm{~nm}$ in the present case) and whose symmetry is more or less commensurate to the bilayer excursions, one should be able to observe geometrydependent effects.

Once the nucleation point is created, fast spreading of the vesicle onto the surface indicates the onset of regime

\footnotetext{
(27) Prost, J .; Manneville, J .-B.; Bruinsma, R. Eur. Phys. J . B 1998,
} $1,465$.
II. It occurs at constant surface and vol ume of the vesicle. In the case of anisotropic patterns such as stripes, the spreading devel ops faster al ong the stripeaxis than along the corresponding in-plane perpendicular axis. Indeed, although spreading is limited in both cases by the drainage of a thin aqueous film, a zip process takes place al ong the stripe axis whereas jumps are required along the perpendicular direction. 25,28,29 Despitetheexpected difference of kinetics and in marked contrast with regime III, the shape of thevesicleremains spherical at the end of regime II. It is also interesting to note that in the time scale investigated in regime II, the absence of optical contrast in EWIF microscopy between the stripes of different attractiveness for the bilayer underlines the absence of

(28) Sandre, O.; Gorre-Talini, L.; Ajdari, A.; Prost, J .; Silberzan, P. Phys. Rev. E 1999, 60, 2964.

(29) Drelich, J .; Wilbur, J . L.; Miller, J . D.; Whitesides, G. M Langmuir 1996, 12, 1913. 

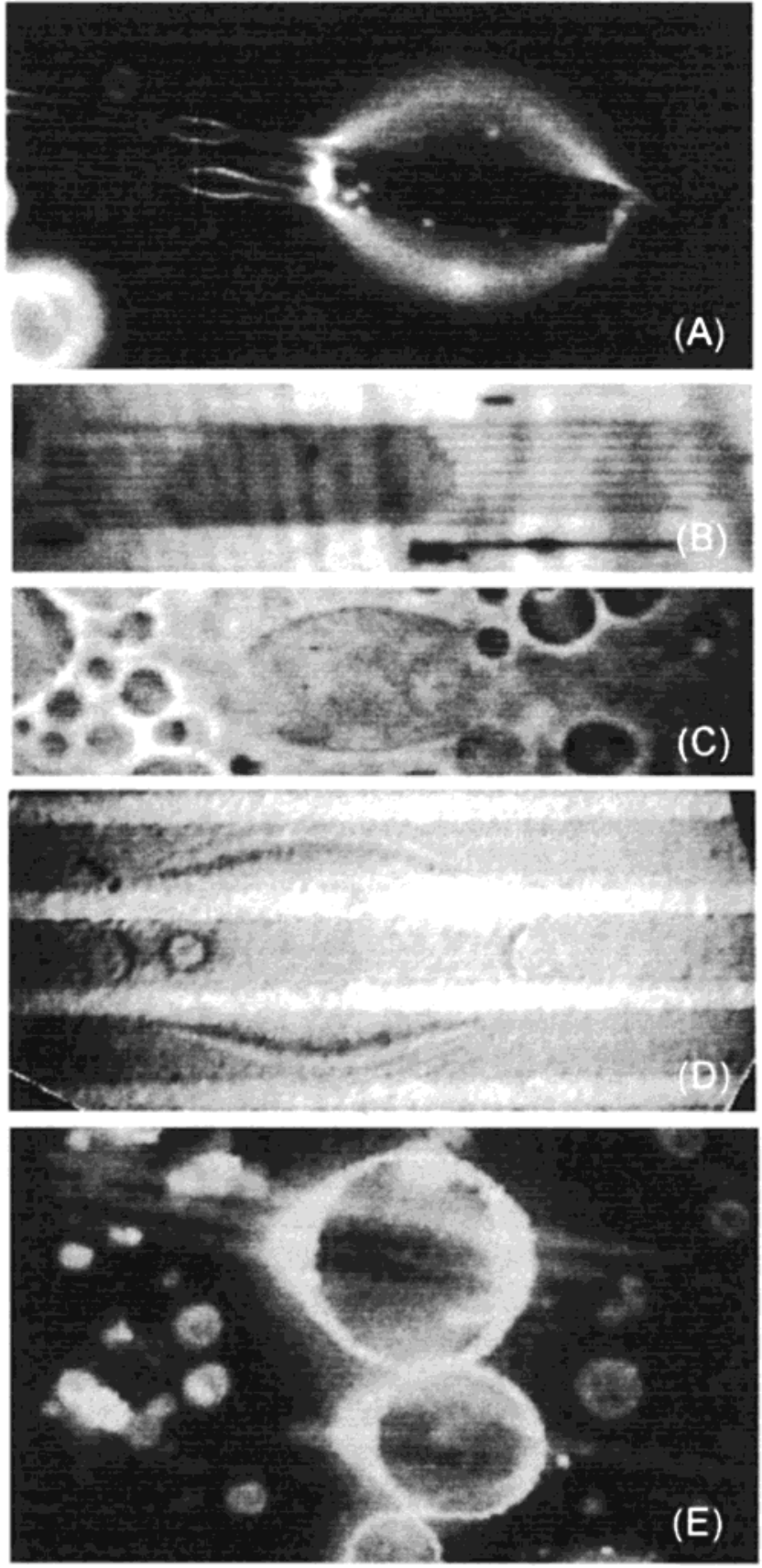

Figure 6. Typical shape of an EPC vesiclespreading on striped patterns ( $2.5 \mu \mathrm{m}$ width) of contrasted attraction as observed in epifluorescence microscopy (A), EWIF microscopy (B), phase contrast microscopy (C), RICM (D); projection of 30 fluorescence cross sections in epifluorescence microscopy; please note the curvature of the membrane induced by the pattern $(E)$.

differences in the thicknesses of the aqueous film located between the surface and the bilayer. Due to the strong adhesion energy, a rather large tension is exerted on the bilayer at the end of regime II. ${ }^{30}$

(30) In the case of smooth surfaces, the adhesion energy W has been evaluated to $(1-5) \times 10^{-3} \mathrm{~mJ} / \mathrm{m}^{2}$ from the consideration of the charge densities of thelipid bilayer and of the surface. Such a valueis generally considered to correspond to a strong adhesion. The corresponding surface tension $\Sigma$ is related to $W$ and to the contact angle of the vesicle on the surface, $\theta$, by the Young-Dupré equation (see: Seifert, U.; Lipowsky, R. Phys. Rev. A 1990, 42, 4768. Nardi, J .; Bruinsma, R.; Sackmann, E. Phys. Rev. E. 1998, 58, 6340.): $W=\Sigma(1-\cos \theta)$. In the present system, the bilayer surface tension at the end of regime $I I$ is evaluated to $5 x$ $10^{-3} \mathrm{mN} / \mathrm{m}$ from both experimental observations and theoretical predictions. See ref 13.
Regime III starts with the release of the corresponding tension by the formation of a macroscopic pore with a radius $r$ that can be estimated as follows. The volume of the nonfluorescent solution expelled from the vesicle between $t=67 \mathrm{~s}$ and $t=68 \mathrm{~s}$ (panels $\mathrm{F}$ and $\mathrm{G}$ of Figure 2) corresponds to a volumic flow J of about $10^{4} \mu \mathrm{m}^{3} \cdot \mathrm{s}^{-1}$ (assuming a spherical dispersion of the vesicle contents). The Laplace pressure drop responsible for this flow J is

$$
\Delta \mathrm{P}=2 \Sigma / \mathrm{R}
$$

where $\Sigma$ is the membranetension and $\mathrm{R}$, thevesicleradius that can be deduced from Figure 2A. If we consider that the flow J through the pore is limited by the viscous dissipation in the aqueous medium (this is justified by an a posteriori Reynolds number that gives $\operatorname{Re} \approx 10^{-3}$ ), we have

$$
\mathrm{J} \approx \Delta \mathrm{Pr}^{3} / \eta
$$

where $\eta$ is the solution viscosity. We have assumed that the pressure drop $\Delta \mathrm{P}$ relaxes on a length $\mathrm{r}$. Taking $\Sigma=$ $5 \times 10^{-3} \mathrm{mN} / \mathrm{m}^{13} \mathrm{R}=40 \mu \mathrm{m}$ (Figure $2 \mathrm{~A}$ ) and $\eta=10^{-3}$ $\mathrm{Pa} \cdot \mathrm{s}$, we obtain a pore radius $\mathrm{r} \approx 5 \mu \mathrm{m}$. This estimate compares with therepeat distance of the decorated pattern and is larger than the recent observed radii of the membrane pores formed under different conditions. ${ }^{31}$ Because of the leakage caused by the pore formation, an excess of area becomes available. Buds form and tubular pseudopods now spread. ${ }^{32}$ In the case of the $2.5 \mu$ m periodic stripe pattern, the spreading stops once enough tension is rel eased. I n contrast, for someother patterns (triangles, squares and herringbone), the spreading vesicles blow up before releasing enough tension to close the pores.

Toproposea model for the dynamics of vesiclespreading during the regime III on all the decorated surfaces tested experimentally is out of the scope of the present paper. Nevertheless, we bring semiquantitative arguments for thestripped pattern. In ref 33, theauthors study the profile of an adhering vesicle on a solid plate in the vicinity of a pinning center. ${ }^{33}$ The latter exerts locally a force $\mathrm{f}_{0}$ on the bilayer. To make the analogy with the adhesive stripes, wewrite $f_{0}=W a$, whereW is the adhesi on energy per unit area and $a$ is the stripe width. The membrane resists against the deformation with an effective line tension $\tau$ given by the relation (see Figure 1)

$$
\tau=\frac{1}{2} \frac{\kappa_{\mathrm{c}}}{\mathrm{R}_{\mathrm{c}}^{2}} \lambda
$$

where $\kappa_{\mathrm{c}}$ is the bending modulus, $\mathrm{R}_{\mathrm{c}}$ is the radius of curvature close to the adhesion area, and $\lambda$ is theangular correlation length that was first introduced in ref 34. It arises from the requirement for the membrane to merge smoothly with thesurface (in contrast with a liquid droplet that makes a sharp wedge). This length is written as $\lambda=$ $\left(\kappa_{d} / \Sigma\right)^{1 / 2}$ and can be measured by RICM. ${ }^{35}$ By estimating

(31) Sandre, O., Moreaux, L., Brochard-Wyart. Proc. Natl. Acad. Sci U.S.A. 1999, 96, 10591.

(32) Thereported coupl ing between water permeation and spreading provides a possiblemechanism which might account for cell motility on surfaces.

(33) Albersdörfer, A.; Bruinsma, R.; Sackmann, E. Europhys. Lett. 1998, 42, 227.

(34) Servuss, R. M.; Helfrich, W. J . Phys. (Paris) 1989, 50, 809.

(35) Albersdörfer, A.; Bruinsma, R.; Sackmann, E. Biophys. J . 1997 

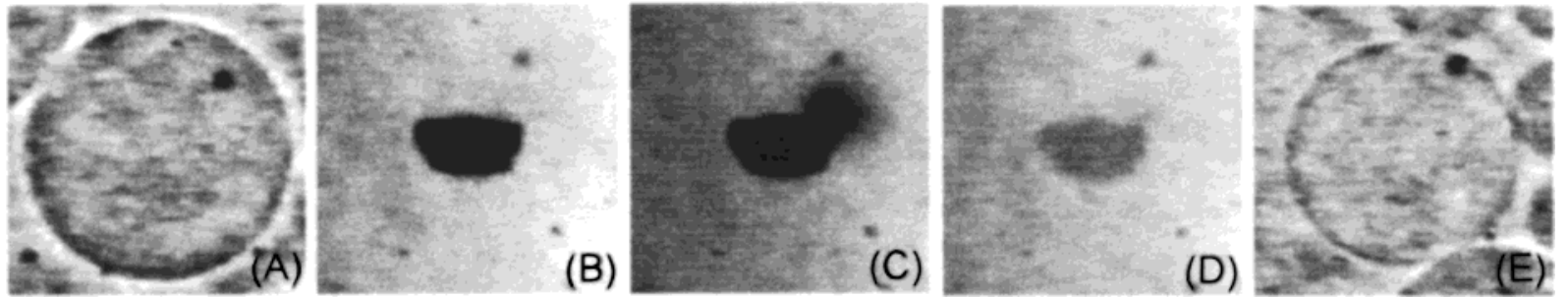

Figure 7. EPC vesicle exhibiting a periodic opening-closing process of a pore during spreading on aged $2.5 \mu \mathrm{m}$ stripe patterns. Vesicle observed in phase contrast microscopy before adhesion (A). Same vesicle observed in EWIF microscopy after adhesion (B C). The periodic opening-closing process ( $0.3 \mathrm{~s}$ duration, $3 \mathrm{~s}$ periodicity) allows the leakage of the fluorescent inner medium of the vesicle (D). Vesicle observed in phase contrast microscopy at the end of the phenomenon (E).
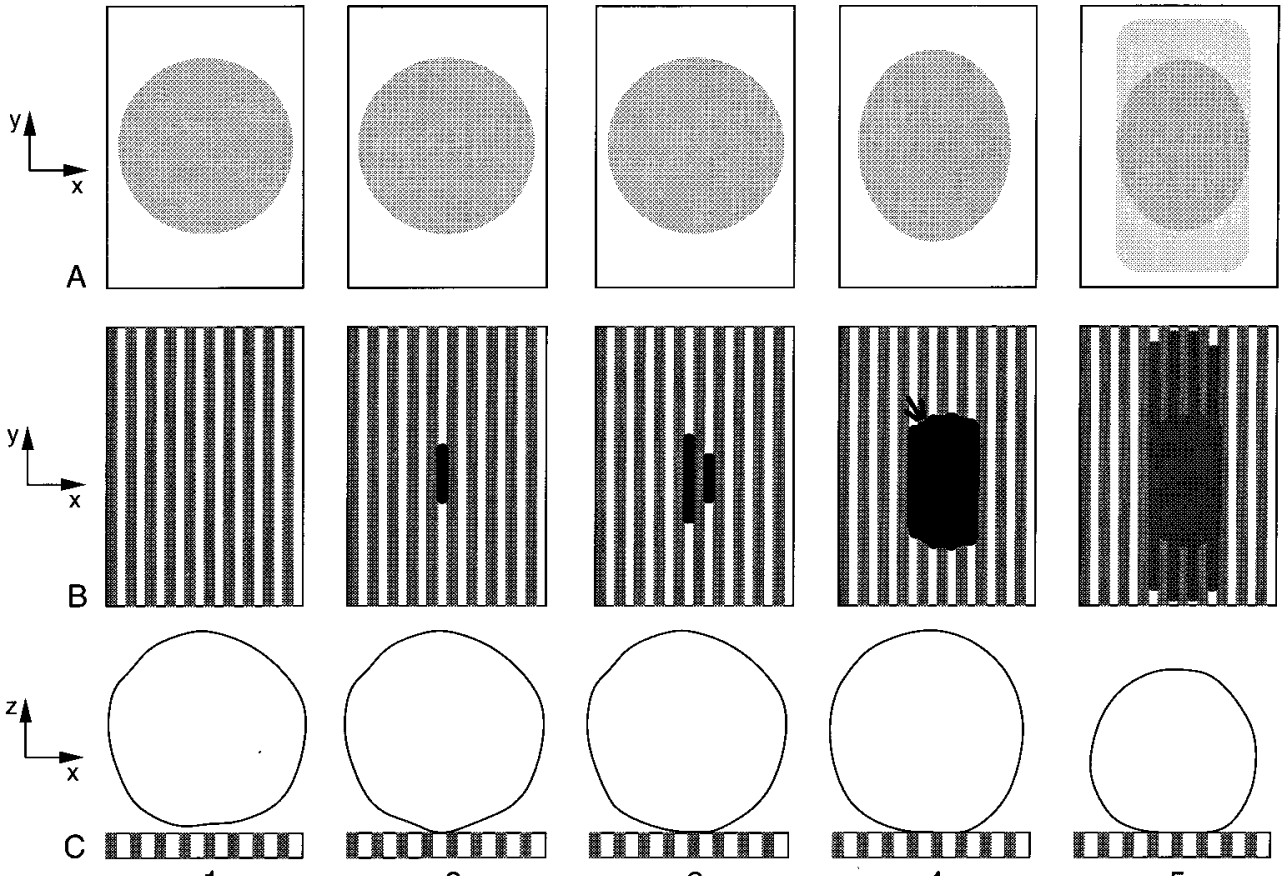

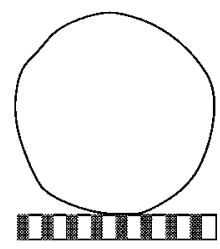

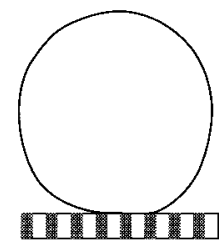

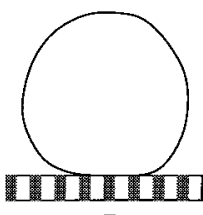

5

Figure 8. Spreading/permeation mechanism of an EPC vesicle on striped patterns ( $2.5 \mu \mathrm{m}$ width) as a function of time (from left to right): (1) regime l, approach; ( 2 and 3 ) regime II, spreading at constant lipid surface but increased contact area and constant volume, no permeation; (4) regime III, formation of a macroscopic pore, spreading at constant lipid surface, the internal volume decreases; (5) equilibrium vesicular shape, the macroscopic pore is closed. (A) Vesicle image in phase contrast microscopy. (B) Vesicle image by EWIF microscopy. (C) Vesicle profile.

also $\mathrm{R}_{\mathrm{c}} \approx \lambda / \theta_{\mathrm{c}}$, where $\theta_{\mathrm{c}}$ is the contact angle, one obtains

$$
\tau=\frac{1}{2} \Sigma \theta_{c}^{2} \lambda
$$

As sketched in Figure 1, the bilayer adopts locally a triangular shape as long as $2 \tau>f_{0}$. In contrast, if the bilayer cannot oppose a sufficient line tension to the applied force, an instability was predicted: $:^{33}$ a tether is pulled from the vesicle. In our experiments, $\Sigma \approx 5 \times 10^{-6}$ $\mathrm{N} \cdot \mathrm{m}^{-1}, 30 \theta_{\mathrm{c}} \approx 15^{\circ}, \mathrm{W}=\Sigma\left(1-\cos \theta_{\mathrm{c}}\right) \approx 10^{-7} \mathrm{~N} \cdot \mathrm{m}^{-1}, \kappa_{\mathrm{c}} \approx$ $4 \times 10^{-20},{ }^{36} \lambda \approx 10^{-7} \mathrm{~m}$, and $2 \tau=2 \times 10^{-14} \mathrm{~N}$. Thelatter estimateis 1 order of magnitudesmaller than the pulling force $f_{0}=3 \times 10^{-13} \mathrm{~N}$. Thus we conclude that the growth of cylindrical protrusions from a vesicle can effectively be driven by spreading of thebilayer onto thecharged stripes of thesubstrate. Sincetheinitially floppy vesicle becomes tenseand adopts a spherical cap shapeat theend of regime II, the growth of the tethers during regime III would lead to dramatically increase the membrane tension $\Sigma$ due to the corresponding increase of the contact area. Hence if therewas nomechanismtoreleasethemembranetension, the tether instability would stop immediately. In fact the

(36) Evans, E.; Rawicz, W. Phys. Rev. Lett. 1990, 64 (17), 2094. process of vesicle deflation through experimentally observed pores keeps long enough a low surface tension to create long protusions.

In ref 31, only vesicles that slowly release a highly viscous sol ution can bear transient pores as large as those estimated in thepresent paper (in a low viscosity medium, both the outflow and the tension release occur too fast to let the pores grow). A possibleexplanation for the present observations is that the pores nucleate at a larger initial radius $r_{c}$ on the decorated surface, where the pattern provides germs above $1 \mu \mathrm{m}$. The nucleation radius is also strongly dependent on the line energy of the bilayer $(\gamma \approx$ $10^{-11} \mathrm{~N}$ for an EPC bilayer) since $\mathrm{r}_{\mathrm{c}}=\gamma / \Sigma .^{37}$ We have invoked the presence of minor amounts of somehydrolysis products in our phosphol ipidic vesicles toaccount for their strong adhesion on oppositely charged surfaces. ${ }^{13}$ Among them, the water-soluble lysolecithins with a single hydrocarbon tail decrease $\gamma$ by a factor of 2 at concentrations as low as $3 \times 10^{-4} \mathrm{mg} / \mathrm{mL}$. ${ }^{38}$ Thus $\mathrm{r}_{\mathrm{c}} \approx 1 \mu \mathrm{m}$ could be

(37) Taupin, C.; Dvolaitzky, M.; Sauterey, C. Biochemistry. 1975, 14 (21), 4768.

(38) Chernomordik, L. V.; Kozlov, M. M.; Melikyan, G. B.; Abidor, I. G.; Markin, V. S.; Chizmadzhev, Y. A. Biochim. Biophys. Acta 1985, 812,643 
expected. Then, the chemical heterogeneities of the solid surface could easily nucleate pores.

\section{Conclusion}

The present work demonstrates that the adhesion of giant vesicles onto decorated surfaces can lead to the permeation of large hydrophilic solutes through lipid bilayers. The observations made by EWIF microscopy on giant vesicles directly demonstrate both the internalization of calcein (size $\approx 1 \mathrm{~nm}$ ) and thecreati on of a transient macroscopic pore (diameter $\approx 1 \mu \mathrm{m}$ ) as a result of the spreading process. I $n$ addition tothesenew and interesting features, some selectivity for transport might beexpected. In fact, thesizeand the lifetime of the pore could probably be tuned by conveniently tailoring the size and the geometry of the patterns as well as the relative surface fractions $x_{s}$ of the decorated surfaces.

Acknowledgment. This study has been supported by L'Oréal. Wegreatly acknowledgeP rofessor Sackmann for the access to RICM facilities and Dr. V. Marchi-Artzner for her assistance during preliminary RICM measure ments. Wethank Dr. Vanlerberghefor fruitful discussions.

DOI: 10.1021/LA991342P 